\title{
Lower Rydberg series of methane: A combined coupled cluster linear response and molecular quantum defect orbital calculation
}

\author{
A. M. Velasco \\ Departamento de Química Fisica y Química Inorgánica, Facultad de Ciencias, \\ Universidad de Valladolid, 47005 Valladolid, Spain
}

\author{
J. Pitarch-Ruiz, Alfredo M. J. Sánchez de Merás, and J. Sánchez-Marín ${ }^{a)}$ \\ Universitat de València, Institut de Ciència Molecular, Edificio de Institutos, \\ Apartado de Correos 22085, E-46071, Valencia, Spain \\ I. Martin \\ Departamento de Química Fisica y Química Inorgánica, Facultad de Ciencias, \\ Universidad de Valladolid, 47005 Valladolid, Spain
}

(Received 21 October 2005; accepted 30 January 2006; published online 27 March 2006)

\begin{abstract}
Vertical excitation energies as well as related absolute photoabsorption oscillator strength data are very scarce in the literature for methane. In this study, we have characterized the three existing series of low-lying Rydberg states of $\mathrm{CH}_{4}$ by computing coupled cluster linear response (CCLR) vertical excitation energies together with oscillator strengths in the molecular-adapted quantum defect orbital formalism from a distorted $C_{s}$ geometry selected on the basis of outer valence green function calculations. The present work provides a wide range of data of excitation energies and absolute oscillator strengths which correspond to the Rydberg series converging to the three lower ionization potential values of the distorted methane molecule, in energy regions for which experimentally measured data appear to be unavailable. (C) 2006 American Institute of Physics.
\end{abstract}

[DOI: $10.1063 / 1.2179069]$

\section{INTRODUCTION}

The structure and physicochemical behavior of $\mathrm{CH}_{4}$ has attracted the attention of scientists for several good reasons. Methane is the most abundant of the minor constituents in the upper atmospheres of the outer planets, and is the dominant continuous photoabsorber in the above atmospheres in the energy region comprised between 8 and $14 \mathrm{eV}^{1-3}$ The observational data confirm that $\mathrm{CH}_{4}$ is present in auroral emissions. ${ }^{4-6}$ Nevertheless, for a better understanding of the spectroscopic and collisional properties of this molecule at various temperatures and under the influence of different perturbers, new experimental and theoretical investigations are clearly needed.

Methane is a natural gas emitted from areas such as rice cultures, forests, and farms, and also from industrial areas and gas pipelines. Despite its natural origin, a growing number of sources for this compound are man made nowadays. Thus, such hydrocarbon is increasingly present in the atmosphere, but it usually reacts away very rapidly with $\mathrm{OH}$ radicals. As a consequence, its influence on the Earth's climate is much less severe than that of fluorinated hydrocarbons. On the other hand, electron collisions with hydrocarbon molecules are important in many other fields of science and technology, ranging from astrophysics ${ }^{7}$ to semiconductor processing. ${ }^{8}$ For instance, in almost all currently operating fusion processes, plasma-wall interactions are an abundant source of hydrocarbon molecules that contaminate the hydro-

\footnotetext{
${ }^{a)}$ Author to whom all correspondence should be addressed. Electronic mail: jose.sanchez@uv.es
}

genic plasma. The composition of hydrocarbon fluxes entering the plasma covers a wide spectrum of molecules, from $\mathrm{CH}_{4}$ to $\mathrm{C}_{3} \mathrm{H}_{8}$.

Due to its small size and simple electronic structure, $\mathrm{CH}_{4}$ has been the subject of extensive theoretical and experimental studies. The accurate determination of its ionization and fragmentation energies is an important basis for the understanding of many electron-impact phenomena. In particular, most of the high-energy chemical processes are initiated by the interaction of medium-energy electrons $(5-50 \mathrm{eV})$ with matter.

Methane is a representative example of a highly symmetrical molecule affected by the Jahn-Teller effect. If one of the six $1 t_{2}$ electrons of $\mathrm{CH}_{4}$ is vertically excited into a Rydberg state, the resultant $\mathrm{CH}_{4}^{+}$ion core suffers a strong JahnTeller distortion and can be considered to remain in a high vibrational state: the tetrahedral structure of neutral methane becomes distorted in the lowest state of methanium cation.

The photoionization peak of the six electrons in the $1 t_{2}$ valence orbital is spread out between 12.5 and $16 \mathrm{eV} .{ }^{10}$ The center of gravity of this broad peak lies approximately at $14.25 \mathrm{eV}$, almost $2 \mathrm{eV}$ beyond the adiabatic ionization potential (IP) of $\mathrm{CH}_{4}$. The first band in the photoelectron spectrum of $\mathrm{CH}_{4}$, as recently determined by Kimura et al. ${ }^{11}$ with photoelectron spectroscopy, is the terminating member of a Rydberg series, and displays three maxima, at 13.6, 14.4, and $15.0 \mathrm{eV}$, respectively. The vibrational envelope of this band is described as resulting from the Jahn-Teller splitting of the degenerate state of the ion. ${ }^{12-16}$

Other several studies ${ }^{10,12,17}$ indicate that a distortion of 
TABLE I. Exponents and coefficients for the ANO Rydberg functions of $\mathrm{CH}_{4}$.

\begin{tabular}{|c|c|c|c|c|c|c|}
\hline \multirow{3}{*}{$\begin{array}{l}\text { Exponents } \\
0.024624\end{array}$} & \multicolumn{6}{|c|}{ Coefficients } \\
\hline & \multicolumn{5}{|c|}{$s$ functions } & \multirow[b]{2}{*}{-3.46560536} \\
\hline & 0.64346061 & -0.48466219 & 0.39871924 & -2.64713664 & 2.52564954 & \\
\hline 0.011253 & 0.40434998 & -0.71209019 & 0.77259787 & 4.17678724 & -7.58420682 & 14.91407209 \\
\hline 0.005858 & -0.01054290 & 0.86564309 & -1.54239222 & 0.22848777 & 8.39195896 & -33.04669903 \\
\hline 0.003346 & -0.01384978 & 0.70902879 & -1.66463645 & -3.31289840 & 0.33097111 & 42.95871438 \\
\hline 0.002048 & -0.01861979 & 0.12574029 & 1.82327338 & -0.54145775 & -6.72079184 & -30.25621275 \\
\hline 0.001324 & 0.01859431 & -0.10636966 & 0.69109847 & 2.40988942 & 0.71688650 & 9.33515966 \\
\hline 0.000893 & -0.00835644 & 0.05279937 & 0.07206496 & -0.57774320 & 2.96653748 & -2.51719634 \\
\hline 0.000624 & 0.00206017 & -0.01389529 & -0.00617252 & 0.20240310 & -0.18719214 & 2.17417374 \\
\hline \multicolumn{7}{|c|}{$p$ functions } \\
\hline 0.042335 & 0.26098976 & -0.29898413 & 0.40637381 & -0.95353256 & 1.16858995 & -2.45645222 \\
\hline 0.019254 & 0.57327290 & -0.52602765 & 0.36393925 & 0.24023370 & -1.35011820 & 7.38301597 \\
\hline 0.009988 & 0.17382854 & 0.18384395 & -0.64742547 & 1.78430766 & -1.64258813 & -11.72109195 \\
\hline 0.005689 & 0.12460477 & 0.57777835 & -0.82945180 & -0.63905671 & 4.45029711 & 11.31883281 \\
\hline 0.003476 & -0.10792932 & 0.44611157 & 0.10369269 & -1.84598861 & -1.34261308 & -8.37682352 \\
\hline 0.002242 & 0.08896236 & -0.01730935 & 0.85749445 & 0.34083552 & -2.40201036 & 9.57926408 \\
\hline 0.001511 & -0.05055929 & 0.04452417 & 0.33172639 & 0.54543757 & -0.59266940 & -11.00003088 \\
\hline 0.001055 & 0.01368673 & -0.01384282 & 0.08091209 & 0.60743136 & 2.22669551 & 5.23289381 \\
\hline \multicolumn{7}{|c|}{$d$ functions } \\
\hline 0.060540 & 0.07634620 & -0.07562858 & 0.11685713 & -0.56634660 & 1.09555918 & -1.58774739 \\
\hline 0.027446 & 0.26801670 & -0.25064394 & 0.28843555 & -0.60015000 & -0.84909189 & 3.57192964 \\
\hline 0.014204 & 0.39919353 & -0.32899450 & 0.13517816 & 0.67310242 & -1.04504225 & -3.71394649 \\
\hline 0.008077 & 0.29944315 & -0.05366972 & -0.02261253 & 1.02654202 & 1.47393879 & 1.04436450 \\
\hline 0.004927 & 0.09106297 & 0.42734295 & -1.05702676 & -0.80179431 & 0.40122659 & 0.39757760 \\
\hline 0.003175 & 0.01448592 & 0.47279316 & 0.44954794 & -0.24150591 & 0.61888430 & 3.56647761 \\
\hline 0.002137 & -0.00155880 & 0.18414058 & -0.71228886 & -1.16806516 & -4.08317352 & -7.14440967 \\
\hline 0.001491 & 0.00044697 & 0.03054713 & 1.51055422 & 1.43937321 & 2.88612495 & 3.79240033 \\
\hline
\end{tabular}

$\mathrm{CH}_{4}^{+}$from the tetrahedral configuration to a $D_{2 d}$ geometry is energetically favored. The $13.6 \mathrm{eV}$ maximum may be interpreted as due to a ${ }^{2} B_{2}$ state in the $D_{2 d}$ structure. ${ }^{10}$ Then, the molecule would be deformed in a $C_{2 v}$ fashion so as to incipiently produce $\mathrm{CH}_{2}^{+}$and $\mathrm{H}_{2}$, and in a $C_{3 v}$ fashion to lead to $\mathrm{CH}_{3}^{+}+\mathrm{H}^{18}$ These predissociation processes into $\mathrm{CH}_{3}^{+}+\mathrm{H}$ and $\mathrm{CH}_{2}^{+}+\mathrm{H}_{2}$ occur at 14.4 and $15.0 \mathrm{eV}$, respectively. ${ }^{19,20}$

Three Koopmans' IPs can be obtained from a single calculation on neutral methane if an appropriately distorted geometry is selected. Therefore, we have found more useful ways to determine a suitable geometry that facilitates to assign the series of Rydberg states out of the calculated vertical excitation energies (VEEs) and the corresponding ionization potentials than performing a detailed study of the vibronic

TABLE II. Displacement vector applied to the experimental $T_{d}$ geometry of $\mathrm{CH}_{4}$ and the cartesian coordinates. All values are given in angstroms for the distorted geometry ( $C_{s}$ symmetry group). See text for details.

\begin{tabular}{|c|c|c|c|c|c|c|}
\hline \multirow[b]{2}{*}{ Atoms } & \multicolumn{3}{|c|}{$\begin{array}{c}\text { Displacement vector } \\
{\left[Q_{3 b}\left(t_{2}\right) \text { mode }\right]}\end{array}$} & \multicolumn{3}{|c|}{ Coordinates } \\
\hline & $X$ & $Y$ & $Z$ & $X$ & Y & $Z$ \\
\hline $\mathrm{C} 1$ & 0.06 & -0.07 & 0 & 0 & 0 & 0 \\
\hline $\mathrm{H} 2$ & 0.01 & -0.01 & 0 & -0.627879 & 0.888520 & -0.000015 \\
\hline H3 & 0.02 & -0.01 & -0.01 & -0.627879 & -0.888520 & -0.000015 \\
\hline $\mathrm{H} 4$ & -0.40 & -0.40 & 0.41 & 0.576691 & 0 & -0.771545 \\
\hline H5 & -0.40 & 0.40 & -0.42 & 0.675812 & 0 & 1.005769 \\
\hline
\end{tabular}

couplings associated to the excitation to low-lying Rydberg states. To determine the VEEs, we have adopted a response function approach with a coupled cluster reference function. This wave function guarantees a size-extensive treatment of the dynamic correlation and ensures an accurate description of those systems characterized by a single-reference description. Together with a subsequent linear response calculations, this method provides good theoretical estimations of secondorder properties and has satisfactorily been applied to the calculation of VEEs and IPs. $^{21,22}$

Once the required Rydberg series and ionization energies were identified, one-photon transition intensities were calculated with the molecular-adapted quantum defect orbital (MQDO) approach. $^{23}$ This theoretical procedure has been also previously used successfully to compute oscillator strengths for transitions involving molecular Rydberg states of different compounds of relevance in the Earth's atmosphere and in astrophysical regions. ${ }^{24,25}$

TABLE III. Valence vertical ionization potentials (in eV) of $\mathrm{CH}_{4}$.

\begin{tabular}{lcccc}
\hline \hline Ionization process & Koopmans' process $^{\mathrm{a}}$ & OVGF $^{\mathrm{b}}$ & EOM-CCSD $^{\mathrm{c}}$ & Expt. $^{\mathrm{d}}$ \\
\hline $\mathrm{CH}_{4} \rightarrow \mathrm{CH}_{4}^{+}+e^{-}$ & $\left(4 a^{\prime}\right)^{-1}$ & 13.62 & 13.813 & 13.6 \\
$\mathrm{CH}_{4} \rightarrow \mathrm{CH}_{3}^{+}+\mathrm{H}+e^{-}$ & $\left(1 a^{\prime \prime}\right)^{-1}$ & 14.21 & 14.421 & 14.4 \\
$\mathrm{CH}_{4} \rightarrow \mathrm{CH}_{2}^{+}+\mathrm{H}_{2}+e^{-}$ & $\left(3 a^{\prime}\right)^{-1}$ & 14.80 & 15.008 & 15.0 \\
\hline \hline
\end{tabular}

${ }^{\mathrm{a}}$ As described in the distorted $C_{s}$ geometry. See text for details.

${ }^{\mathrm{b}}$ This work. $C_{s}$ distorted geometry. Basis set $6-31 \mathrm{G}(2 d, 1 p)$.

${ }^{\mathrm{c}}$ This work. $C_{s}$ distorted geometry. Basis set $[5 s 4 p 3 d 1 f / 4 s 3 p 1 d+6 s 6 p 6 d]$. ${ }^{\mathrm{d}}$ Kimura et al. (Ref. 11). 
TABLE IV. Vertical excitation energies $(\mathrm{eV})$ for the $\mathrm{IP}=14.421 \mathrm{eV}$ of $\mathrm{CH}_{4}$.

\begin{tabular}{cccccc}
\hline \hline & \multicolumn{3}{c}{$T_{d}$} & & \multicolumn{2}{c}{$C_{s}$} \\
\cline { 2 - 3 } \cline { 5 - 6 } State & CCSD & CCSD(3) & & CCSD & $\operatorname{CCSDR}(3)$ \\
\hline $3 s$ & 10.63 & 10.59 & & 10.61 & 10.57 \\
$4 s$ & 12.81 & 12.78 & & 12.82 & 12.79 \\
$3 p$ & 11.84 & 11.80 & & 11.88 & 11.84 \\
$4 p$ & 13.18 & 13.15 & & 13.17 & 13.13 \\
$3 d \mathrm{II}^{\mathrm{a}}$ & 12.71 & 12.67 & & 12.81 & 12.77 \\
$4 d \mathrm{II}^{\mathrm{a}}$ & 13.46 & 13.43 & & 13.51 & 13.48 \\
$3 d \mathrm{I}^{\mathrm{b}}$ & 12.64 & 12.60 & & 12.70 & 12.67 \\
$4 d \mathrm{I}^{\mathrm{b}}$ & 13.43 & 13.40 & & 13.47 & 13.44 \\
\hline \hline
\end{tabular}

${ }^{\mathrm{a}} \mathrm{II}=x^{2}-y^{2}$.

${ }^{\mathrm{b}} \mathrm{I}=x z, y z$.

\section{COMPUTATIONAL DETAILS}

Green function based methods can provide fairly improved IPs that include the Dyson self-energy effective potential evaluated perturbatively up to third order plus renormalization terms starting from the Koopmans' IPs as zeroorder approach. In particular, the outer valence green function (OVGF) method supplies correlated values of the outer valence IPs at a moderate computational cost. Therefore, the outer valence IPs were calculated within the OVGF approach in a systematic exploration of a set of deformed geometries constructed using the SCF/6-31G(2d,p) normal coordinates.

Vertical excitation energies were computed inside a coupled cluster linear response formalism using a linked triples corrected CCSD wave function as reference function, ${ }^{26-28}$ with the effect of connected triples estimated by means of the CCSDR(3) method. ${ }^{29}$ For systems that can be properly described by simple reference methods, triples corrected coupled cluster methods give excitation energies that are correct to third order in the fluctuation potential. ${ }^{29}$ As coupled cluster of singles and doubles linear response (CCSDLR) provides exactly the same excitation energies than the equation of motion coupled cluster of singles and doubles (EOM-CCSD) method, this last approximation was used to compute the IPs at the CCSD level. The $1 s$ core orbitals were kept frozen in all the coupled cluster calculations.

We have used an atomic natural orbitals (ANO) basis $\operatorname{set}^{30}$ with contractions $[5 s 4 p 2 d 1 f]$ and $[4 s 3 p 1 d]$ for carbon and hydrogen, respectively. It has been shown in a previous work $^{31}$ that this basis delivers essentially the same results than Dunning's correlation consistent aug-cc-pVTZ basis. In addition, the ANO set has been augmented with a series of $6 s 6 p 6 d$ Rydberg functions allocated on the carbon atom and built by following the technique proposed by Roos et al. to generate a universal Gaussian basis set. ${ }^{32}$ Table I shows the coefficients and exponents obtained for the Rydberg function basis set.

The EOM calculations were carried out with the ACESII (Ref. 33) code, and the other coupled cluster calculations with the use of the DALTON program, ${ }^{34}$ in which the abovementioned algorithms are implemented. GAUSSIAN 03 (Ref. 35) was used for the OVGF calculations as well as for the SCF geometry optimization.
TABLE V. Energy levels in $\mathrm{eV}$ and quantum defects $(\delta)$ for the Rydberg series of $\mathrm{CH}_{4}$ corresponding to the $D_{2 d}$ symmetry $(\mathrm{IP}=13.813 \mathrm{eV})$.

\begin{tabular}{|c|c|c|c|c|c|c|}
\hline \multirow[b]{2}{*}{ State } & \multicolumn{5}{|c|}{$E / \mathrm{eV}$} & \multirow{2}{*}{$\frac{\delta}{\operatorname{CCSDR}(3)^{\mathrm{d}}}$} \\
\hline & Expt. $^{\mathrm{a}}$ & Expt. $^{\text {b }}$ & Expt. $^{\mathrm{c}}$ & Theor. $^{\mathrm{a}}$ & $\operatorname{CCSDR}(3)^{\mathrm{d}}$ & \\
\hline $3 s a_{1}$ & 9.70 & 9.698 & 9.70 & 9.70 & 10.02 & 1.11 \\
\hline $4 s a_{1}$ & 11.80 & 11.54 & 11.61 & 11.95 & 12.20 & 1.10 \\
\hline $5 s a_{1}$ & & & & & 12.91 & 1.12 \\
\hline $6 s a_{1}$ & & & & & 13.23 & 1.17 \\
\hline $3 p_{x, y} e$ & & & & & 11.25 & 0.70 \\
\hline $4 p_{x, y} e$ & & & & & 12.56 & 0.70 \\
\hline $5 p_{x, y} e$ & & & & & 13.07 & 0.72 \\
\hline $6 p_{x, y} e$ & & & & & 13.31 & 0.80 \\
\hline $3 p_{z} b_{2}$ & & & & & 11.31 & 0.67 \\
\hline $4 p_{z} b_{2}$ & & & & & 12.61 & 0.64 \\
\hline $5 p_{z} b_{2}$ & & & & & 13.08 & 0.69 \\
\hline $6 p_{z} b_{2}$ & & & & & 13.33 & 0.69 \\
\hline $3 d_{z^{2}} a_{1}$ & 12.1 & & & 12.09 & 12.09 & 0.19 \\
\hline $4 d_{z^{2}} a_{1}$ & & & & & 12.82 & 0.30 \\
\hline $5 d_{z^{2}} a_{1}$ & & & & & 13.16 & 0.44 \\
\hline $3 d_{x^{2}-y^{2}} b_{1}$ & & & & & 12.02 & 0.25 \\
\hline $4 d_{x^{2}-y^{2}} b_{1}$ & & & & & 12.83 & 0.28 \\
\hline $5 d_{x^{2}-y^{2}} b_{1}$ & & & & & 13.18 & 0.36 \\
\hline $3 d_{x y} b_{2}$ & & & & & 11.99 & 0.27 \\
\hline $4 d_{x y} b_{2}$ & & & & & 12.81 & 0.32 \\
\hline $5 d_{x y} b_{2}$ & & & & & 13.16 & 0.44 \\
\hline $3 d_{x z, y z} e$ & & & & & 12.19 & 0.10 \\
\hline $4 d_{x z, y z} e$ & & & & & 12.89 & 0.16 \\
\hline $5 d_{x z, y z} e$ & & & & & 13.22 & 0.21 \\
\hline
\end{tabular}

${ }^{\mathrm{a}} \mathrm{Au}$ et al. (Ref. 42).

${ }^{b}$ Lee and Chiang (Ref. 43).

${ }^{\mathrm{c}}$ Robin (Ref. 44)

${ }^{\mathrm{d}}$ This work.

The MQDO method was formulated for calculating intensities of transitions involving Rydberg states. ${ }^{23}$ In the MQDO formalism a model, one-electron Hamiltonian with a parametric potential is employed. The radial quantum defect orbitals are analytical solutions of the corresponding Schrödinger equation. The angular counterparts are linear combinations of spherical harmonics chosen so that the complete MQDOs form basis functions for the different irreducible representations of the pertinent molecular symmetry group.

\section{RESULTS AND DISCUSSION}

\section{A. Selection of the geometry}

The threefold degeneracy of the $t_{2}$ valence molecular orbital (MO) makes it difficult a simple assignment of each state in the vertical manifold of excitations to a particular Rydberg series converging to one of the three outer valence IPs of $\mathrm{CH}_{4}$. However, by removing such degeneracy, one can obtain three estimated valence IPs that can be unambiguously assigned to each of the three highest occupied MO on the basis of Koopmans' theorem. ${ }^{36}$ Then, a given state can be simply assigned by inspection of the hole MO in the excita- 
TABLE VI. Energy levels in $\mathrm{eV}$ and quantum defects $(\delta)$ for the Rydberg series $\mathrm{of}_{\mathrm{CH}}$ corresponding to the $C_{3 v}$ symmetry $(\mathrm{IP}=14.421 \mathrm{eV})$.

\begin{tabular}{|c|c|c|c|c|c|c|c|}
\hline \multirow[b]{2}{*}{ State } & \multicolumn{6}{|c|}{$E / \mathrm{eV}$} & \multirow{2}{*}{$\frac{\delta}{\operatorname{CCSDR}(3)^{\mathrm{e}}}$} \\
\hline & Expt. $^{a}$ & Expt. $^{b}$ & Expt. $^{\mathrm{c}}$ & Theor. $^{\text {a }}$ & Theor. $^{\mathrm{d}}$ & $\operatorname{CCSDR}(3)^{\mathrm{e}}$ & \\
\hline $3 s a_{1}$ & 10.50 & 10.41 & 10.37 & 10.50 & 10.59 & 10.57 & 1.12 \\
\hline $4 s a_{1}$ & 12.8 & & & 12.75 & 12.64 & 12.79 & 1.11 \\
\hline $5 s a_{1}$ & & & & & 13.34 & 13.50 & 1.16 \\
\hline $6 s a_{1}$ & & & & & & 13.82 & 1.24 \\
\hline $3 p_{x, y} e$ & & & & 12.04 & 11.66 & 11.84 & 0.70 \\
\hline $4 p_{x, y} e$ & & & & & 12.98 & 13.13 & 0.75 \\
\hline $5 p_{x, y} e$ & & & & & & 13.66 & 0.77 \\
\hline $6 p_{x, y} e$ & & & & & & 13.92 & 0.79 \\
\hline $3 p_{z} a_{1}$ & & & & 12.04 & 11.66 & 11.89 & 0.68 \\
\hline $4 p_{z} a_{1}$ & & & & & 12.98 & 13.21 & 0.65 \\
\hline $5 p_{z} a_{1}$ & & & & & & 13.66 & 0.77 \\
\hline $6 p_{z} a_{1}$ & & & & & & 13.91 & 0.84 \\
\hline $3 d_{z^{2}} a_{1}$ & & & & & 12.62 & 12.68 & 0.20 \\
\hline $4 d_{z^{2}} a_{1}$ & & & & & 13.32 & 13.31 & 0.50 \\
\hline $5 d_{z^{2}} a_{1}$ & & & & & & 13.72 & 0.59 \\
\hline $3 d_{x^{2}-y^{2}, x y} e \mathrm{I}$ & & & & 12.75 & $12.62-12.63$ & 12.67 & 0.21 \\
\hline $4 d_{x^{2}-y^{2}, x y} e \mathrm{I}$ & & & & 13.49 & $13.32-13.33$ & 13.44 & 0.28 \\
\hline $5 d_{x^{2}-y^{2}, x y} e \mathrm{I}$ & & & & & & 13.80 & 0.32 \\
\hline $3 d_{x z, y z} e \mathrm{II}$ & 12.9 & & & 12.89 & 12.66 & 12.77 & 0.13 \\
\hline $4 d_{x z, y z} e \mathrm{II}$ & 13.5 & & & 13.55 & 13.33 & 13.48 & 0.20 \\
\hline $5 d_{x z, y z} e \mathrm{II}$ & & & & & & 13.81 & 0.28 \\
\hline
\end{tabular}

${ }^{\mathrm{a}} \mathrm{Au}$ et al. (Ref. 42).

${ }^{\mathrm{b}}$ Lee and Chiang (Ref. 43).

${ }^{\mathrm{c}}$ Robin (Ref. 44).

${ }^{\mathrm{d}}$ Cacelli et al. (Ref. 41).

${ }^{\mathrm{e}}$ This work.

tions representing it. The main drawback of this approach is that a direct correlation to a particular structure of the $\mathrm{CH}_{4}^{+}$ cation with specific symmetry resulting from the Jahn-Teller effect ${ }^{37}$ coupled to the ionization process is not possible.

Clearly the easiest way to accomplish the removal of degeneracy is distorting the geometry of the highly symmetrical $T_{d}$ structure of methane. With this aim, we have deformed the experimental geometry $\left(R_{\mathrm{CH}}=1.087 \AA\right)$ along those normal coordinates that break completely the threefold degeneracy. Therefore the modes $\nu_{4 a}\left(t_{2}\right), \nu_{3 c}\left(t_{2}\right)$, and $\nu_{2 a}(e)$ were discarded. From the other modes, a set of distorted geometries $R$ were built according to $R=R_{0}+k Q_{i}$, where $R_{0}$ stands for the vector of experimental nuclear coordinates at equilibrium, $k$ is a scaling factor, and $Q_{i}$ denotes the vector of mass-weighted Cartesian displacement corresponding to the $i$ normal coordinate. At each geometry $R$, valence IPs were calculated with the OVGF approach, ${ }^{38,39}$ to minimize the mean absolute error of calculated IPs with respect to experiment.

The best mean absolute error in the OVGF IP values was obtained with the $\nu_{3 b}\left(t_{2}\right)$ normal coordinate, using a value of $k=0.20$. The components of the selected $Q_{i}$ vector, as well as the final geometry, of $C_{s}$ symmetry, adopted in the present calculations, are given in Table II.

\section{B. Ionization potentials and transition energies}

The calculated ionization potentials, together with the more recent experimental values, reported by Kimura et al. ${ }^{11}$ are displayed in Table III. We have also included the dissociative ionization processes associated with each IP. The EOM-CCSD results reported in Table III have been used along with the CCLR values in the calculation of the quantum defects.

In order to have a general view of the effect of the geometry distortions on the excitations we present, in Table IV, some of the calculated energies (i.e., those for the Rydberg series that converges to $14.421 \mathrm{eV}$ ). As can be observed, there are no important differences between the energy values obtained, independently if we consider $T_{d}$ or $C_{s}$ symmetry. Note, however, that the three series would be degenerate in the $T_{d}$ case. It seems also apparent in Table IV that inclusion of linked triples in the CCSDR(3) calculations, diminishes the magnitude of all excitation energies in approximately $0.02-0.03 \mathrm{eV}$. Therefore, given the small difference between the two sets of excitation energies and the systematic nature of the triples correction, we have only considered the CCSDR(3) results in the remaining of this section.

The presently calculated VEEs, together with some of the few existing comparative values, are displayed in Tables V-VII. Accordingly to the pioneering work of Mulliken, ${ }^{40}$ 
TABLE VII. Energy levels in $\mathrm{eV}$ and quantum defects $(\delta)$ for the Rydberg series of $\mathrm{CH}_{4}$ corresponding to the $C_{2 v}$ symmetry $(\mathrm{IP}=15.008 \mathrm{eV})$.

\begin{tabular}{|c|c|c|c|}
\hline \multirow[b]{2}{*}{ State } & \multicolumn{2}{|c|}{$E / \mathrm{eV}$} & \multirow{2}{*}{$\frac{\delta}{\operatorname{CCSDR}(3)^{\mathrm{b}}}$} \\
\hline & Expt. $^{a}$ & $\operatorname{CCSDR}(3)^{\mathrm{b}}$ & \\
\hline $3 s a_{1}$ & $11.11^{\mathrm{c}}$ & 11.14 & 1.12 \\
\hline $4 s a_{1}$ & & 12.96 & 1.42 \\
\hline $5 s a_{1}$ & & 14.09 & 2.15 \\
\hline $6 s a_{1}$ & & 14.41 & 2.23 \\
\hline $3 p_{x} b_{1}$ & & 12.41 & 0.71 \\
\hline $4 p_{x} b_{1}$ & & 13.71 & 0.76 \\
\hline $5 p_{x} b_{1}$ & & 14.25 & 0.76 \\
\hline $6 p_{x} b_{1}$ & & 14.49 & 0.87 \\
\hline $3 p_{y} b_{2}$ & & 12.44 & 0.7 \\
\hline $4 p_{y} b_{2}$ & & 13.75 & 0.71 \\
\hline $5 p_{y} b_{2}$ & & 14.25 & 0.76 \\
\hline $6 p_{y} b_{2}$ & & 14.50 & 0.82 \\
\hline $3 p_{z} a_{1}$ & & 12.47 & 0.68 \\
\hline $4 p_{z} a_{1}$ & & 13.75 & 0.71 \\
\hline $5 p_{z} a_{1}$ & & 14.25 & 0.76 \\
\hline $6 p_{z} a_{1}$ & & 14.49 & 0.87 \\
\hline $3 d_{z^{2}, x^{2}-y^{2}} a_{1}$ & & 13.28 & 0.19 \\
\hline $4 d_{z^{2}, x^{2}-y^{2}} a_{1}$ & & 14.04 & 0.25 \\
\hline $5 d_{z^{2}, x^{2}-y^{2}} a_{1}$ & & 14.38 & 0.35 \\
\hline $3 d_{x y} a_{2}$ & & 13.18 & 0.27 \\
\hline $4 d_{x y} a_{2}$ & & 13.96 & 0.40 \\
\hline $5 d_{x y} a_{2}$ & & 14.33 & 0.52 \\
\hline $3 d_{x z} b_{1}$ & & 13.35 & 0.14 \\
\hline $4 d_{x z} b_{1}$ & 14.1 & 14.07 & 0.19 \\
\hline $5 d_{x z} b_{1}$ & & 14.4 & 0.27 \\
\hline $3 d_{y z} b_{2}$ & & 13.36 & 0.13 \\
\hline $4 d_{y z} b_{2}$ & 14.1 & 14.07 & 0.19 \\
\hline $5 d_{y z} b_{2}$ & & 14.4 & 0.27 \\
\hline
\end{tabular}

${ }^{\mathrm{a}} \mathrm{Au}$ et al. (Ref. 42).

${ }^{\mathrm{b}}$ This work.

${ }^{c}$ Extrapolated using a quantum defect of 1.13 .

we have written the $n l$ atomic orbital notation corresponding to atomic neon (the united-atom limit of $\mathrm{CH}_{4}$ ), with which the ending $\mathrm{MO}$ in a given molecular electron excitation may be correlated. The $n l$ nature associated with the Rydberg MOs was determined by inspecting the nodal structure and the magnitude of the quantum defect of each of the atomic orbitals found in the dominant configuration of the molecular excited states. The quantum defects have also been included in the tables, together with the irreducible representation symbol, $\Gamma_{i}$, to which the states belong. This notation is commonly used because the motion of the outermost electron in a Rydberg state is almost independent of the state of the inner ion. The polarization effects on the inner ion due to the outermost electron are rather small and may not affect the geometrical structure of the molecule. That is, the potential surfaces of the Rydberg series can be considered to follow that of the corresponding ionized state.

To the best of our knowledge, the most extensive $a b$ initio calculation reported on this problem is the one by
Cacelli et al., ${ }^{41}$ who carried out a selected singles and doubles configurations interaction using a rather limited basis set. Cacelli et al. ${ }^{41}$ used the one-center expansion (OCE) approximation, with which they performed all their calculations in the fixed experimental ground-state geometry, within the static exchange frozen orbital approximation. A vertical IP closer to the $C_{3 v}$ ionization band was adopted.

Our results conform well with the theoretical values reported by Cacelli et al., ${ }^{41}$ and also with the experimental data available in the literature. ${ }^{42-44} \mathrm{Au}$ et $a$ al $^{42}$ performed electron energy loss measurements using dipole $(e, e)$ spectroscopy, and also predicted transition energies using the Rydberg formula. They calculated the $n s, n p$, and $n d$ excitation energies using a quantum defect for the $n s$ Rydberg series equal to 1.13 , and assuming average values for $\delta_{p}$ and $\delta_{d}$ equal to 0.6 and 0.0 , respectively. In a similar way, in Table VII we have included a new energy value for the $3 s$ Rydberg state, that has been obtained by employing the same quantum defect as Au et al., ${ }^{42}$ equal to 1.13 . The so-obtained energy value for the $3 s$ Rydberg state is in good agreement with our calculations.

However, there exist significant deviations between two of our computed excitation energies with respect to experiment. In particular, the $3 s$ and $4 s$ states, which converge to the lowest IP, (see Table V) exhibit errors of the order of 0.3-0.4 eV, unlike the expected error of around $0.1 \mathrm{eV}$. These large deviations reflect the fact that the selected distorted geometry may not be the optimal one for these states, but, in a sense, this is a price to pay to get an overall good description of the whole set of quantum defects.

Inspection of any of the Tables V-VII reveals that in analogous Rydberg series, as are the ones under study, important similarities in the magnitude of the quantum defects occur, in particular, for the first members of each Rydberg series. This feature may be explained on the grounds of assuming that the term values, $t=E-\mathrm{IP}$, are transferable from one IP limit to another in transitions occurring within different Rydberg series, in this context. This is most likely due to the highly nonbonding and diffuse nature of the Rydberg orbitals, on to which the molecular core acts nearly as a point charge.

\section{MQDO oscillator strengths}

The oscillator strengths for all the allowed transitions of the present distorted form of $\mathrm{CH}_{4}$, originating in the ground state (GS) and ending in a Rydberg state have been collected in Table VIII. In the table, the transitions have been grouped according to the IP to which the higher-lying Rydberg states converge.

The electron configuration of the ${ }^{1} A_{1}$ ground state of $\mathrm{CH}_{4}$ in its $T_{d}$ tetrahedral form is $\left(1 a_{1}\right)^{2}\left(2 a_{1}\right)^{2}\left(1 t_{2}\right)^{6}$, where the $1 t_{2} \mathrm{MO}$ may be correlated to the $2 p \mathrm{AO}$ of the united atom limit, $\mathrm{Ne}$ atom. ${ }^{45}$ Given the $2 p$ character of the leastbound electron in the GS, ${ }^{45}$ only transitions to states with $n s$ or $n d$ character are listed. It is a well-known fact that those molecular Rydberg transitions that obey Laporte's selection rule are much stronger than otherwise.

The notation used in Table VIII for the GS (i.e., the 
TABLE VIII. Oscillator strengths for different Rydberg electronic transitions from the ground state of $\mathrm{CH}_{4}$.

\begin{tabular}{|c|c|c|c|c|c|}
\hline \multicolumn{2}{|c|}{$D_{2 d}$} & \multicolumn{2}{|c|}{$C_{3 v}$} & \multicolumn{2}{|c|}{$C_{2 v}$} \\
\hline Transition & MQDO & Transition & MQDO & Transition & MQDO \\
\hline $1 b_{2} \rightarrow 3 s a_{1}$ & 0.0943 & $3 a_{1} \rightarrow 3 s a_{1}$ & 0.0893 & $3 a_{1} \rightarrow 3 s a_{1}$ & 0.0808 \\
\hline $1 b_{2} \rightarrow 4 s a_{1}$ & 0.0173 & $3 a_{1} \rightarrow 4 s a_{1}$ & 0.0166 & $3 a_{1} \rightarrow 4 s a_{1}$ & 0.0248 \\
\hline $1 b_{2} \rightarrow 5 s a_{1}$ & 0.0068 & $3 a_{1} \rightarrow 5 s a_{1}$ & 0.0070 & $3 a_{1} \rightarrow 5 s a_{1}$ & 0.0079 \\
\hline $1 b_{2} \rightarrow 6 s a_{1}$ & 0.0036 & $3 a_{1} \rightarrow 6 s a_{1}$ & 0.0038 & $3 a_{1} \rightarrow 6 s a_{1}$ & 0.0032 \\
\hline $1 b_{2} \rightarrow 3 d a_{1}$ & 0.0387 & $3 a_{1} \rightarrow 3 d a_{1}$ & 0.0357 & $3 a_{1} \rightarrow 3 d a_{1}$ & 0.0310 \\
\hline $1 b_{2} \rightarrow 4 d a_{1}$ & 0.0211 & $3 a_{1} \rightarrow 4 d a_{1}$ & 0.0273 & $3 a_{1} \rightarrow 4 d a_{1}$ & 0.0157 \\
\hline $1 b_{2} \rightarrow 5 d a_{1}$ & 0.0126 & $3 a_{1} \rightarrow 5 d a_{1}$ & 0.0208 & $3 a_{1} \rightarrow 5 d a_{1}$ & 0.0093 \\
\hline $1 b_{2} \rightarrow 3 d e$ & 0.0456 & $3 a_{1} \rightarrow 3 d e \mathrm{II}$ & 0.0430 & $3 a_{1} \rightarrow 3 d b_{1}, b_{2}$ & 0.0387 \\
\hline $1 b_{2} \rightarrow 4 d e$ & 0.0240 & $3 a_{1} \rightarrow 4 d e \mathrm{II}$ & 0.0198 & $3 a_{1} \rightarrow 4 d b_{1}, b_{2}$ & 0.0208 \\
\hline $1 b_{2} \rightarrow 5 d e$ & 0.0135 & $3 a_{1} \rightarrow 5 d e \mathrm{II}$ & 0.0138 & $3 a_{1} \rightarrow 5 d b_{1}, b_{2}$ & 0.0122 \\
\hline
\end{tabular}

initial state in the transitions) is that of the outermost MO in the $D_{2 d}, C_{3 v}$, or $C_{2 v}$ symmetry group, that is, $1 b_{2}, 3 a_{1}$, or $3 a_{1}$, respectively, as done before. An inspection of the table reveals the same order of magnitude for those oscillator strengths that belong to analogous Rydberg series among those sets that converge to different IPs. This feature is easily explained on the grounds of the close similarities in the quantum defects that characterize the above different Rydberg series. Following the argument given in the last paragraph of the precedent section, the term values, $t=E-\mathrm{IP}$, are transferable from one IP to another of the Rydberg transitions' limits, with the consequence that the intensity of the analogous transitions belonging to different Rydberg series are very much alike. It may be worth mentioning that transition probabilities from the GS to the degenerate pair of $n d b_{1}$ and $n d b_{2}$ Rydberg states in the $C_{2 v}$ structure, i.e., the one converging to the third of the IPs considered in this work, have been added up and collected in the pertinent location of Table VIII.

Table IX displays the absolute MQDO oscillator strengths obtained after integration in different energy ranges comprised between 8.55 to about $11 \mathrm{eV}$, given the experimental fact that, as a careful analysis reveals, in these energy

TABLE IX. Integrated oscillator strengths of $\mathrm{CH}_{4}$.

\begin{tabular}{|c|c|c|}
\hline Transitions & $\mathrm{MQDO}^{\mathrm{a}}$ & Expt. \\
\hline $\left.\begin{array}{l}1 b_{2} \rightarrow 3 s a_{1} \\
3 a_{1} \rightarrow 3 s a_{1} \\
3 a_{1} \rightarrow 3 s a_{1}\end{array}\right\}$ & 0.2644 & $\begin{array}{c}0.277 \pm 0.0355^{\mathrm{b}} \\
0.36 \pm 0.04^{\mathrm{c}} \\
0.29 \pm 0.03^{\mathrm{d}} \\
0.26^{\mathrm{e}} \\
0.39 \pm 0.04^{\mathrm{f}} \\
0.27 \pm 0.03^{\mathrm{g}} \\
0.29^{\mathrm{h}} \\
0.425^{\mathrm{i}}\end{array}$ \\
\hline
\end{tabular}

${ }^{\mathrm{a}}$ This work.

${ }^{\mathrm{b}}$ Harshberger and Lassettre (Ref. 46).

${ }^{\mathrm{c}}$ Edwards and Raymonda (Ref. 51).

${ }^{\mathrm{d}}$ Sun and Weissler (Ref. 47).

${ }^{\mathrm{e}}$ Watanable et al. (Ref. 49).

${ }^{\mathrm{f}}$ Moe and Duncan (Ref. 50).

${ }^{\mathrm{g}}$ Ditchburn (Ref. 48).

${ }^{\mathrm{h}}$ Robin (Ref. 44).

${ }^{\mathrm{i}}$ Lee and Chiang (Ref. 43). ranges more than one transition takes place. More specifically, those intervals comprise the transitions from the ground state to the three Jahn-Teller components of the $3 \mathrm{~s}$ Rydberg state, as indicated in Table IX. Addition of the three individual MQDO oscillator strength values is required for a comparison with the experimental data. A few comments on the experimental techniques that have helped supply the comparative $f$ values are in order.

Harshbarger and Lassettre ${ }^{46}$ found, in an electron impact experiment, the integrated oscillator strength within the $8.55-10.95 \mathrm{eV}$ energy region. The $f$ values reported by these authors have also been attributed to an integration of the absorption coefficients obtained in earlier optical absorption studies by Sun and Weissler, ${ }^{47}$ by Ditchburn, ${ }^{48}$ and by Watanable et al., ${ }^{49}$ through an analogous integration procedure to the one used in their electron impact spectra. The data quoted from Moe and Duncan ${ }^{50}$ and from Edwards and Raymonda $^{51}$ in Table IX are the oscillator strengths determined, according to their own authors, by deconvoluting the overlapping bands of the first and second transitions. Moe and Duncan, ${ }^{50}$ from measurements over the $8.55-11.27 \mathrm{eV}$ entire region, warned the likely presence of a large error in the experimental results due to unresolved rotational fine structure. The reported absolute intensities by Edwards and Raymonda, ${ }^{51}$ from vacuum ultraviolet spectra, achieved absolute intensities that should be reliable, according to the own authors, to $25 \%$. An integrated oscillator strength of 0.425 was measured by Lee and Chiang ${ }^{43}$ with synchrotron radiation covering the $8.73-11.697 \mathrm{eV}$ energy region. Finally, Robin ${ }^{44}$ integrated the energy loss in methane from 8.55 to $10.95 \mathrm{eV}$, which yielded an oscillator strength of 0.29 .

An overall inspection of Table IX makes apparent the general similarities of the presently calculated oscillator strengths with the scarce experimental values that are, to our knowledge, available. We associate this fact to the good quality of the VEEs and IPs achieved in a previous step of this work with accurate $a b$ initio calculations, as well as to the undoubtedly correct assignment of the resulting manifold of Rydberg states in the $C_{s}$ distorted geometry of $\mathrm{CH}_{4}$ to the three Rydberg series that converge, respectively, on each of the three different IPs presently calculated. 


\section{CONCLUSIONS}

Complete series of Rydberg states and transitions converging to each of the three different valence IP values exhibited by a selected distorted geometry of the neutral methane molecule have been calculated. Through coupled cluster approaches, a wide manifold of transitions, that presented a seriously difficult individual assignment to the very few existing experimental data, was obtained. The scarcity of experimental data may be possibly due to the large Jahn-Teller coupling experienced by the $t_{2}^{-1}$ ground state of the $\mathrm{CH}_{4}^{+}$ cation. Our assignments conform satisfactorily well to the scarce data that can be found in the literature. The technique followed in the present work has relied on a careful examination of the quantum defects associated with each of the initial manifold of states, calculated in $C_{s}$ symmetry. Then, by discerning, one by one, the Rydberg states of higher symmetries $\left(D_{2 d}, C_{3 v}\right.$, and $\left.C_{2 v}\right)$, a set of practically constant quantum defects that match all the terms in each $n l$ different Rydberg series was sought. This procedure was applied within the spirit of the MQDO method, which was also further used to calculate absorption oscillator strengths.

In summary, the LR-CCSD and LR-CCSDR(3) VEEs used along the EOM-CCSD calculated values for the IPs, were analyzed on the grounds of the expected limit symmetry of the cation. In addition, the use of the MQDO approach allowed us to perform the assignment of quantum defects to each Rydberg state on the basis of the above $a b$ initio data. As an additional test, we ensured that each of the newly found series of Rydberg states did converge to one of the three calculated IPs.

We may summarize our analysis by remarking that both energy and transition probability indicators in the present calculations show consistency with the values reported from experimental measurements. We take this fact as an assessment of the accuracy of the LR-CCSDR(3) approach and, also, of the adequacy of the MQDO methodology in performing a clear-cut classification of Rydberg states of unknown symmetry. The MQDO methodology has also proved, in a number of molecular studies, to yield good-quality transition intensities.

\section{ACKNOWLEDGMENTS}

This research was supported by Grant No. CHE0135823 from the U.S. National Science Foundation, as well as by the Spanish MEC, Spanish GV, and European FEDER Funds, (Project Nos. CTQ2004-07768-C02/BQU and GVINFRA03-047). One of the authors (A.M.V.) also wishes to acknowledge her research agreement from the same institution.

\footnotetext{
${ }^{1}$ G. H. Mount and H. W. Moos, Astrophys. J. 224, L35 (1978).

${ }^{2}$ B. Carli and M. Carlotti, in Spectroscopy of the Earth's Atmosphere and Interstellar Medium, edited by R. K. Narahari and A. Weber (Academic, New York, 1992).

${ }^{3}$ E. Karkoschka, Icarus 111, 174 (1994).

${ }^{4}$ J. Ajello, D. Shemonsky, W. Prior et al., J. Geophys. Res. 103, 20125 (1998).

${ }^{5}$ W. R. Pryor, K. Ajello, K. Tobiska et al., J. Geophys. Res. 103, 20149 (1998).
}

${ }^{6}$ S. T. Durrance, P. D. Feldman, and H. W. Moss, Geophys. Res. Lett. 9, 652 (1982).

${ }^{7}$ A. Dalgarno, Dissociative Recombination: Theory, Experiment and Applications IV (World Scientific, Singapore, 2000).

${ }^{8}$ V. Tarnovsky and K. Becker, Plasma Sources Sci. Technol. 4, 307 (1995).

${ }^{9}$ R. K. Janev, Comments At. Mol. Phys. 36, 83 (1991).

${ }^{10}$ A. W. Potts and W. C. Price, Proc. R. Soc. London, Ser. A 326, 165 (1972).

${ }^{11}$ K. Kimura, S. Katsumata, Y. Achiba, T. Yamazaki, and S. Iwata, Handbook of He l Photoelectron Spectra of Fundamental Organic Molecules (Japan Scientific Society, Tokyo, 1981).

${ }^{12}$ N. Dixon, Mol. Phys. 20, 113 (1971).

${ }^{13}$ C. R. Brundle, M. B. Robin, and H. Bosh, J. Chem. Phys. 53, 2196 (1970).

${ }^{14}$ B. P. Pullen, T. A. Carlson, W. Moddeman, G. K. Shweitzer, W. E. Bull, and F. A. Grimm, J. Chem. Phys. 53, 768 (1970).

${ }^{15}$ H. J. Lempka, D. G. Strects, A. W. Potts, and W. C. Price, Philos. Trans. R. Soc. London, Ser. A 268, 59 (1970).

${ }^{16}$ J. W. Rabalais, T. Bergmark, L. O. Werme, L. Karlsson, and K. Siegbahn, Phys. Scr. 3, 14 (1971).

${ }^{17}$ F. A. Grimm and J. Godoy, Chem. Phys. Lett. 6, 336 (1970).

${ }^{18}$ S. Karplus and R. Bersohn, J. Chem. Phys. 51, 2040 (1969).

${ }^{19}$ W. A. Chupka, J. Chem. Phys. 48, 2337 (1968).

${ }^{20}$ V. H. Dibeler, M. Krauss, R. M. Rees, and F. N. Harlee, J. Chem. Phys. 42, 379 (1965).

${ }^{21}$ O. Christiansen, H. Koch, P. Jørgensen, and J. Olsen, Chem. Phys. Lett. 256, 185 (1996).

${ }^{22}$ J. F. Stanton and J. Gauss, J. Chem. Phys. 101, 8938 (1994).

${ }^{23}$ I. Martín, C. Lavín, A. M. Velasco, M. O. Martín, J. Karwowski, and G. H. F. Diercksen, Chem. Phys. Lett. 202, 307 (1996).

${ }^{24}$ A. M. Velasco, I. Martín, J. Pitarch-Ruiz, and J. Sanchez-Marín, J. Phys. Chem. A 108, 6724 (2004).

${ }^{25}$ J. V. Ortiz, I. Martín, A. M. Velasco, and C. Lavín, J. Chem. Phys. 120, 7949 (2004).

${ }^{26}$ H. Koch and P. Jørgersen, J. Chem. Phys. 93, 3333 (1990).

${ }^{27}$ O. Christiansen, H. Koch, A. Halkier, P. Jørgersen, T. Helgaker, and A. Sánchez de Merás, J. Chem. Phys. 105, 6921 (1996).

${ }^{28}$ H. Koch, A. Sánchez de Merás, T. Helgaker, and O. Christiansen, J. Chem. Phys. 104, 4157 (1986).

${ }^{29}$ O. Christiansen, J. Kock, and P. Jørgersen, J. Chem. Phys. 103, 7429 (1995).

${ }^{30}$ P. O. Widmark, P. A. Malmqvist, and B. O. Roos, Theor. Chim. Acta 77, 291 (1990).

${ }^{31}$ A. M. Sánchez de Merás, I. García Cuesta, and H. Koch, Chem. Phys. Lett. 348, 469 (2001).

${ }^{32}$ B. O. Roos, M. Fülscher, P. A. Malmqvist, M. Merchán, and L. SerranoAndrés, Theoretical Studies of the Electronic Spectra of Organic Molecules (Kluwer Academic, Dordrecht, 1995).

${ }^{33}$ Computer code ACESII, Advanced Concepts in Electronic Structure II, Quantum Theory Project, Version 0.3 (University of Florida, Gainsville, 1999).

${ }^{34}$ T. Helgaker, H. L. Aa. Jensen, P. Jørgersen et al., computer code DALTON, a molecular electronic structure program. Release 1.2 (2001), see http:// www.kjemi.uio.no/software/dalton/dalton.html.

${ }^{35}$ M. J. Frisch, G. W. Trucks, H. B. Schlegel et al., computer code GAUSsIAN 03 (Gaussian, Pittsburgh, 2003).

${ }^{36}$ A. Koopmans, Physica (The Hague) 1, 104 (1933).

${ }^{37}$ G. Herzberg, Molecular Spectra and Molecular Structure (Van Nostrand, New York, 1966), Vol. II.

${ }^{38}$ J. V. Ortiz, J. Chem. Phys. 89, 6348 (1988).

${ }^{39}$ J. V. Ortiz, Int. J. Quantum Chem., Quantum Chem. Symp. 23, 321 (1989).

${ }^{40}$ R. S. Mulliken, J. Chem. Phys. 3, 517 (1935).

${ }^{41}$ I. Cacelli, V. Carravetta, and R. Moccia, J. Phys. B 18, 1375 (1985).

${ }^{42}$ J. W. Au, G. Cooper, G. R. Burton, T. N. Olney, and C. R. Brion, Chem. Phys. 173, 209 (1993).

${ }^{43}$ L. C. Lee and C. C. Chiang, J. Chem. Phys. 78, 688 (1983).

${ }^{44}$ M. B. Robin, Higher Excited States of Polyatomic Molecules (Academic, New York, 1985), Vol. III.

${ }^{45}$ G. Herzberg, Molecular Spectra and Molecular Structure (Van Nostrand, New York, 1966), Vol. III. 
${ }^{46}$ W. R. Harshbarger and E. N. Lassettre, J. Chem. Phys. 58, 1505 (1973).

${ }^{47}$ H. Sun and G. L. Weissler, J. Chem. Phys. 23, 1160 (1955).

${ }^{48}$ R. W. Ditchburn, Proc. R. Soc. London, Ser. A 229, 44 (1955).

${ }^{49}$ K. Watanable, M. Zelikoff, and E. L. Y. Inn, "Absorption coefficients of several atmosferic gases," Air Force Cambridge Research Center. Technical Report 53-23 (Boston, MA, 1953).

${ }^{50}$ G. Moe and A. B. F. Duncan, J. Am. Chem. Soc. 74, 3140 (1952).

${ }^{51}$ L. Edwards and J. W. Raymonda, J. Am. Chem. Soc. 91, 5937 (1969). 